\title{
The arithmetic of a successful mitral valve repair
}

\author{
Muralidhar Padala, $\mathrm{PhD}$
}

From the Division of Cardiothoracic Surgery, Carlyle Fraser Heart Center, Emory University, Atlanta, Ga.

Disclosures: Author has nothing to disclose with regard to commercial support.

Received for publication July 16, 2017; accepted for publication July 25, 2017; available ahead of print September 6, 2017.

Address for reprints: Muralidhar Padala, PhD, Emory University, Cardiothoracic Surgery, 550 Peachtree St NE,

Cardiothoracic Surgery Research Laboratory, Atlanta, GA 30308 (E-mail: spadala@emory.edu).

J Thorac Cardiovasc Surg 2017;154:1638-40

$0022-5223 / \$ 36.00$

Copyright (c) 2017 by The American Association for Thoracic Surgery

http://dx.doi.org/10.1016/j.jtcvs.2017.07.043

In this issue of the Journal, Yoshida and colleagues ${ }^{1}$ report the use of 320-slice computed tomographic imaging to pre-operatively measure the length of the mitral valve chordae tendineae to repair a flail leaflet. In their technique, the mitral leaflets and the papillary muscle heads are identified in the volumetric computed tomographic images. The distance from the papillary muscle tip to the identical leaflet region on the healthy opposite leaflet is measured (in this case the posterior leaflet) and a neochorda of this length is deployed on the prolapsing anterior leaflet. The proposed concept for mitral repair is equivalent to chordal transposition, which has been used extensively with good outcomes. $^{2,3}$ Use of 320 slice computed tomography is novel, but the need for such high-quality images for such a simple measurement may be farfetched and expensive. Nonetheless, Yoshida and colleagues ${ }^{1}$ report a new technique to measure mitral valve dimensions to aid in a successful mitral repair.

Although the emphasis of this publication is on the use of sophisticated imaging and software tools, the true challenge remains in identifying which preoperative geometric factors to measure and how to integrate these measurements into the surgery. To bridge preoperative information to intraoperative optimization of the technique, the surgeon needs to understand some concepts about mitral valve function.

The first concept is that when mitral valve leaflets close in systole, their rough zones (ie, the leaflet segment between the secondary chordal insertion and the primary chordal insertion) are parallel to each other (Figure 1, A). This parallel alignment of leaflet edges is observed from one commissure to the other, irrespective of the number of chordae or their origin in the papillary muscle heads. This is a result of the anatomic design of the mitral valve, wherein the rough zone of the leaflet acts as a sail that is restrained between the tugging force of the primary and secondary chordae tendineae. The primary chordae are short, stiff, and less extensible, and they thus restrain the leaflet edges more apically in the ventricle during systole. The secondary chordae are longer, less stiff, and more

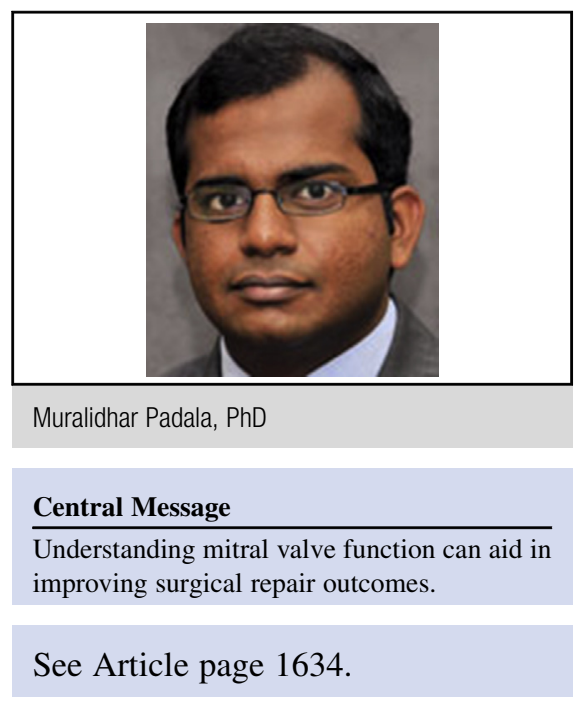

extensible, and they thus allow the remainder of the leaflet to move more basally toward the mitral annulus. As the left ventricular pressure increases, the rough zone captured between the primary and secondary chordae is therefore approximately vertical, whereas the smooth zone between the secondary chordae and the mitral annulus bulges basally. In the setting of mitral valve flail, the primary chordal restraint is completely lost, and thus the sail (ie, the rough zone) everts towards the left atrium and causes regurgitation (Figure 1, B). Neochordoplasty should focus on restoring this primary chordal restraint, such that rough edge parallelization in systole is restored. The optimal length of the neochorda to attain this objective can be determined as shown in Figure 1, $C$. First, the length of the intact secondary chorda from its insertion in the leaflet to its insertion in the papillary muscle head should be measured, defining the maximum allowed length of the neochordal loop $\left(l_{\max }\right)$. Second, the diastolic distance between the leaflet edge and the secondary chordal insertion in the papillary muscle head should be measured, which defines the minimum allowed length of the neochordal loop $\left(l_{\min }\right)$. A value that is at a midpoint between $1_{\min }$ and $1_{\max }$ may be sufficient to achieve leaflet parallel alignment. Theoretically, this value should achieve a coaptation length that is at least half the length of the rough zone of either leaflet. If higher coaptation is required, the chordal loop length can be titrated accordingly, but without exceeding $1_{\max }$. Once the desired length is determined, a goretex loop should be inserted into the papillary muscle at the secondary chordal origin. The loop is then passed through the leaflet edge, and a small clamp (right angled 

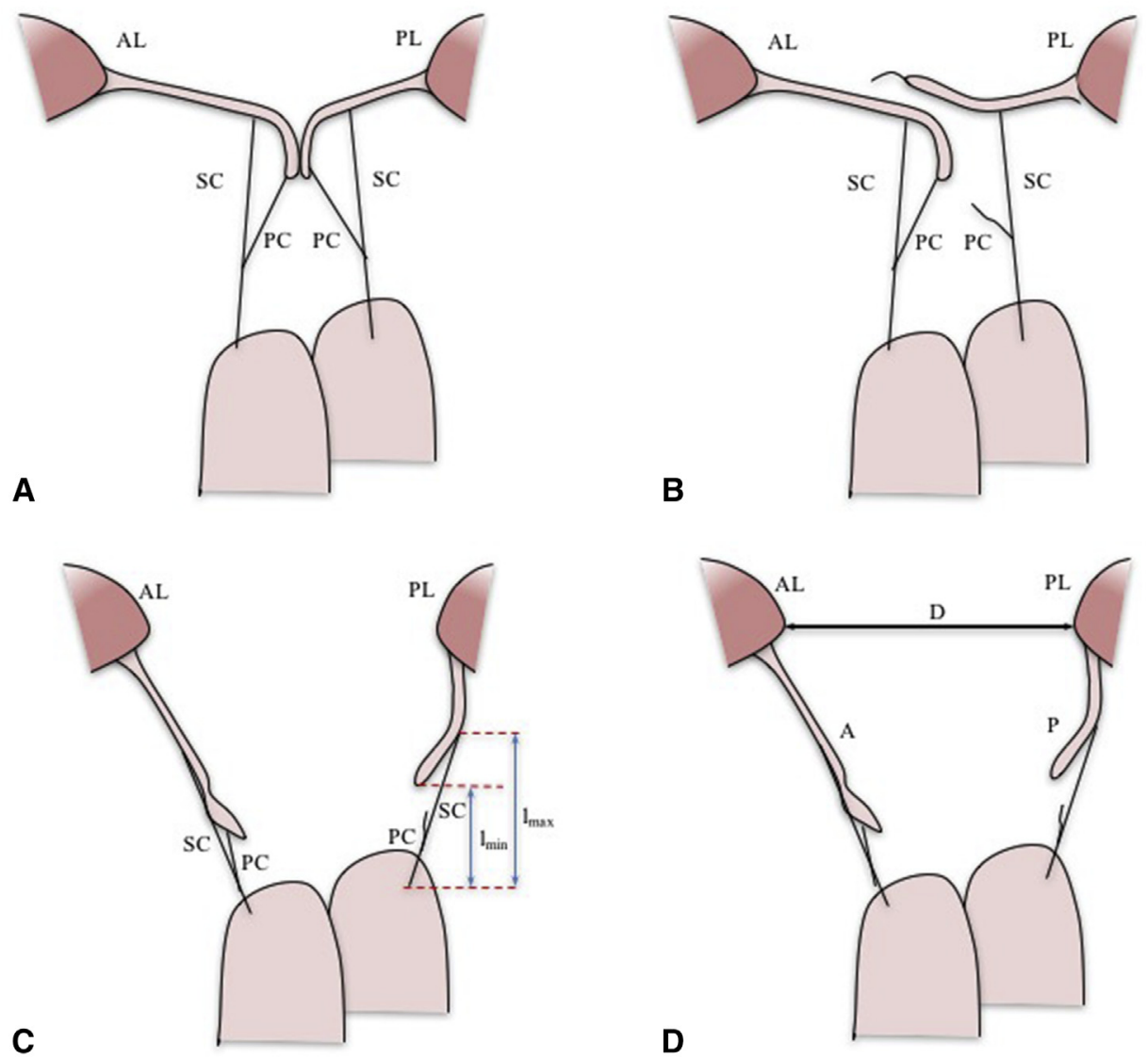

FIGURE 1. A, Schematic of the mitral valve depicting the anterior leaflet $(A L)$ and posterior leaflet $(P L) . P C$ indicates the primary chorda, and $S C$ indicates the secondary chorda, with the leaflet section captured between the chordal insertion points being the rough zone of the leaflet. B, Flail of the rough zone on a leaflet in which the primary chorda is ruptured. C, Diastolic configuration of the same valve, depicting the two measurements of importance for the repair: $l_{\text {max }}$ indicates the length from secondary chordal insertion in the leaflet to the papillary muscle; $l_{\text {min }}$ indicates the length from the free edge of the leaflet to the insertion point of the secondary chorda in the papillary muscle. $\mathrm{D}$, Another view of the diastolic configuration: $D$ represents the septolateral annular diameter; $A$ represents the diastolic length of the anterior leaflet; and $P$ represents the diastolic length of the posterior leaflet.

hemostat will work) should be placed on the loop at the predetermined length from its papillary muscle insertion site. The first assistant should hold this clamp and tug on the papillary muscle, while the surgeon would then tie the knots onto the clamp. This technique ensures that the loop is of the desired length.

The second concept is that the summed heights of the two leaflets need to be always greater than the septolateral dimension of the mitral annulus at the same site on the valve (Figure 1,D). If the sum of the diastolic anterior and posterior leaflet lengths is greater than or equal to the diastolic septolateral diameter, then annular undersizing may not be necessary, although most surgeons feel more comfortable placing a true-sized annuloplasty ring. There is no evidence in the scientific literature that such annular reinforcement is necessary. If the sum is less than the diastolic septolateral diameter, then some degree of annular undersizing may be necessary. This may be achieved with a simple septolateral annular suture, rather than an annuloplasty ring.
By integrating these concepts, the surgeon will not only achieve a competent mitral valve, but will also preserve adequate mobility of the leaflets. If some degree of leaflet tissue resection is necessary, then the surgeon should complete the resection and closure of the site before performing the steps described here. One of the few neochordoplasty techniques to have focused on parallel alignment of leaflet edges, is the technique proposed by Woo and MacArthur, ${ }^{4}$ in which a posterior ventricular anchoring loop is used to repair the flail leaflet. In summary, the focus needs to veer from increasingly sophisticated imaging to obtain mitral valve dimensions towards increased sophistication in using available imaging data by the surgeon.

\section{References}

1. Yoshida S, Toda K, Miyagawa S, Yoshikawa Y, Sawa Y. Preoperative determination of artificial chordae length using 320-slice computed tomographic images. $J$ Thorac Cardiovasc Surg. 2017;154:1634-7.

2. Padala M, Sweet M, Hooson S, Thourani VH, Yoganathan AP. Hemodynamic comparison of mitral valve repair: techniques for a flail anterior leaflet. J Heart Valve Dis. 2014;23:171-6. 
3. Smedira NG, Selman R, Cosgrove DM, McCarthy PM, Lytle BW, Taylor PC, et al. Repair of anterior leaflet prolapse: chordal transfer is superior to chordal shortening. J Thorac Cardiovasc Surg. 1996;112: 287-91; discussion 291-2.
4. Woo YJ, MacArthur JW Jr. Posterior ventricular anchoring neochordal repair of degenerative mitral regurgitation efficiently remodels and repositions posterior leaflet prolapse. Eur J Cardiothorac Surg. 2013;44: 485-9; discussion 489. 